Por Sebastián

Amaya Gallego*, Carmen Sofía Páez

Bonilla* y Diana Milena Vargas Rodríguez **

\section{ESTRÉS EN LOS \\ ESTUDIANTES DE PSICOLOGÍA \\ A CAUSA DEL AISLAMIENTO \\ PREVENTIVO OBLIGATORIO}

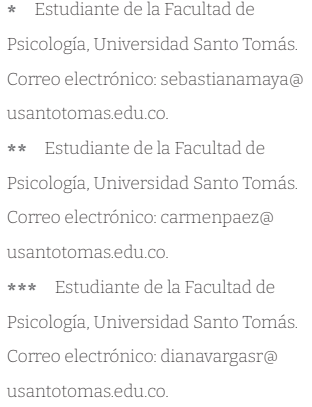

e puede considerar que el estrés no es exclusivamente un evento externo del cual el sujeto es víctima; por el contrario, el estrés psicológico se define como una relación particular entre el individuo y su entorno, en la cual él mismo evalúa la situación como amenazante o desbordante respecto a sus recursos y, por tanto, pone en peligro su bienestar (González y Landero, 2008, p. 8).

Teniendo en cuenta lo anterior, los estímulos negativos, el estrés emocional, la ansiedad o la depresión deterioran las funciones inmunes, provocan una pérdida de la homeostasis-equilibrio y fomentan el desarrollo de patologías. Un estudio como el de Riofrio (2012), cuyo objetivo fue determinar la influencia del estrés agudo en las personas privadas de la libertad del Centro de Rehabilitación Social de la ciudad de Quito, da cuenta de los efectos del aislamiento social y establece que el ingreso a prisión puede ser el inicio de una larga cadena de estímulos estresantes; tales estresores son la ruptura del vínculo social, el desequilibrio emocional, y la situación económica regular, los cuales afectan gravemente la salud mental de la persona. De igual modo, estudios sobre el impacto del aislamiento social en esta población han evidenciado que "esta experiencia es psicológicamente dolorosa, puede ser traumática y nociva, y coloca a muchos de los que han sido sometidos a ella en una situación de riesgo, la cual puede generar daños emocionales e incluso físicos a largo plazo" (Haney y Lynch, 1997, p. 500).

Asimismo, la investigación llevado a cabo por Dorina et al. (2003), en la rama de la salud, evalúa las consecuencias del aislamiento en los cuidadores de pacientes con enfermedades terminales. Según el estudio, 
ellos presentan un notable impacto en el sentimiento de soledad y el estado de aislamiento en general. Así la enfermedad y sus desafíos enfrentan a los cuidadores de dichos pacientes con decisiones urgentes y situaciones límite, lo que probablemente les acarrea un sentimiento de aislamiento existencial. Esta situación es de granimpacto para su salud, ya que los cuidadores no presentan sintomatología o afectaciones en su salud, a diferencia de los pacientes a quienes custodian.

Ahora bien, a la luz de los estudios mencionados anteriormente, la situación actual a nivel mundial es un fenómeno que no habíamos vivido con anterioridad, en especial, por las características que se han adoptado para salvaguardar nuestra salud. La mayoría de la población se ha visto en la obligación de permanecer en aislamiento preventivo en sus hogares a causa de las medidasyestrategias adoptadas por los Gobiernos para controlar la propagación del COVID-19.

Lo cual genera la pregunta por ¿cómo este confinamiento puede llegar a afectar nuestros niveles de estrés? En el contexto colombiano, el aislamiento preventivo rige desde el 18 de marzo con la expedición del Decreto presidencial 457 de 2020. En consecuencia con lo mencionado, el presente estudio cobra gran importancia, ya que a la fecha no existen estudios que evalúen dichos factores (el aislamiento obligatorio y el nivel de estrés) en una población sana como los son los estudiantes universitarios, lo cual da cuenta del vacío académico sobre el tema y su importancia para el estudio del comportamiento humano desde la psicología.

En este ejercicio investigativo participaron 40 estudiantes de psicología de la Universidad Santo Tomás, sede Bogotá, con edades entre 18 y 28 años La media observada fue de 22 y la desviación estándar respectiva de 1.73. En relación con el sexo, participaron 31 mujeres (77.5\%) y 9 hombres (22.5\%). El $30 \%$ de los participantes cursa octavo semestre, el $25 \%$ séptimo semestre, el 22.5\% décimo semestre yel $15 \%$ noveno semestre. Los estudiantes de los semestres más representativos, tercero, cuarto y sexto, participaron con el $2.5 \%$ cada uno, con lo cual todas las medidas de tendencia central(media, mediana y moda) se definieron en el octavo semestre.

Para medir la variable del estrés en esta $\quad 1$ Remor (2001). investigación, se optó por utilizar la escala de estrés percibido (EEP1-10), ya que es la más Conocida en la cuantificación de estrés emocional en investigaciones clínicas y epidemiológicas. Esta posee una fiabilidad (alfa $=.82$, test-retest, $r=.77$ ), validez, y sensibilidad que muestran valores apropiados (6).

Se empleó la versión en castellano de la EEP-10 usada por Remor (2001) en un estudio de validación con adultos en España. Esta escala mide la percepción del estrés psicológico, es decir, la medida en que las situaciones de la vida cotidiana se aprecian como estresantes. La escala incluye una serie de consultas directas que exploran el nivel de estrés experimentado durante el último mes.

La escala brinda cinco opciones de respuesta: nunca, casinunca, devez encuando, muchas veces y siempre, las cuales se clasifican de cero a cuatro. No obstante, los ítems 4, 5, 7 y 8 se puntúan de forma invertida, es decir, de cuatro a cero, ya que estos evalúan la capacidad de afrontamiento de los participantes antelas situaciones estresantes.

Se realizó un análisis estadístico por medio de la herramienta Excel/Spss y se creó una base de datos, en la cual estarán incluidas todas las respuestas de los participantes con las que, luego, se establecieron las medidas de tendencia central y las medidas de dispersión absoluta y relativa, según las características que cumplen las variables. A continuación, se establece la tabla de puntuación (ver tabla 1). 
Tabla 1. Puntuación en la escala de estrés percibido EEP-10.

\begin{tabular}{|c|c|c|c|c|c|}
\hline PUNTUACIÓN EN LA ESCALA DE ESTRÉS PERCIBIDO & NUNCA & $\begin{array}{c}\text { CASI } \\
\text { NUNCA }\end{array}$ & $\begin{array}{l}\text { DE VEZ EN } \\
\text { CUANDO }\end{array}$ & A MENUDO & $\begin{array}{c}\text { MUY A } \\
\text { MENUDO }\end{array}$ \\
\hline $\begin{array}{l}\text { 1. ¿Con qué frecuencia ha estado afectado por algo que ha ocurrido } \\
\text { inesperadamente? }\end{array}$ & O & 1 & 2 & 3 & 4 \\
\hline $\begin{array}{l}\text { 2. ¿Con qué frecuencia se ha sentido incapaz de controlar las cosas } \\
\text { importantes en su vida? }\end{array}$ & O & 1 & 2 & 3 & 4 \\
\hline 3. ¿Con qué frecuencia se ha sentido nervioso o estresado? & O & 1 & 2 & 3 & 4 \\
\hline $\begin{array}{l}\text { 4. ¿Con qué frecuencia ha estado seguro sobre su capacidad } \\
\text { para manejar sus problemas personales? }\end{array}$ & 4 & 3 & 2 & 1 & o \\
\hline 5. ¿Con qué frecuencia ha sentido que las cosas le van bien? & 4 & 3 & 2 & 1 & o \\
\hline $\begin{array}{l}\text { 6. ¿Con qué frecuencia ha sentido que no podía afrontar todas las } \\
\text { cosas que tenía que hacer? }\end{array}$ & $\mathrm{O}$ & 1 & 2 & 3 & 4 \\
\hline 7. ¿Con qué frecuencia ha podido controlar las dificultades de su vida? & 4 & 3 & 2 & 1 & O \\
\hline 8. ¿Con qué frecuencia ha sentido que tenía todo bajo control? & 4 & 3 & 2 & 1 & o \\
\hline $\begin{array}{l}\text { 9. ¿Con qué frecuencia ha estado enfadado porque las cosas } \\
\text { que le han ocurrido estaban fuera de su control? }\end{array}$ & $\mathrm{O}$ & 1 & 2 & 3 & 4 \\
\hline $\begin{array}{l}\text { 10. ¿Con qué frecuencia ha sentido que las dificultades se acumulan } \\
\text { tanto que no puede superarlas? }\end{array}$ & o & 1 & 2 & 3 & 4 \\
\hline
\end{tabular}

Teniendo en cuenta el análisis estadístico realizado sobre los resultados obtenidos en la aplicación de la EEP-10, podemos observar que el $55 \%$ de los datos puntuaron en el nivel moderado de estrés, el $30 \%$ en el nivel bajo y el $15 \%$ restante de la muestra poblacional (40 estudiantes) puntuó en un nivel alto (ver tabla 2). Lo que indica que, a casi 3 meses de confinamiento, el nivel de estrés es tolerable.

Asimismo, las puntuaciones obtenidas enlas preguntas 1, 2, 3, 6, 9 y10 miden de manera directa lapercepción delavariable estrés en elúltimo mes (ver figura 1), en donde el promedio de respuesta estuvo en el rango 2 (de vez en cuando). Por otro lado, los participantes puntuaron en los ítems 4 , 5, 7 y 8 en un rango de 1 y 2 (de vez en cuando y a menudo) (ver figura 2), lo que da cuenta de su capacidad de afrontamiento ante las situaciones estresantes que pudieron vivir en el último mes.

Por último, según las respuestas dadas por los estudiantes en la escala de estrés percibido, se aplicaron las medidas de tendencia central que evidencian una MEDIA de 17.98 puntos, una distribución de los datos 12 y 16 con la misma frecuenciayuna puntuación de 16.5 como medida de posición central. 
Tabla 2. Rango de puntuación en la escala de estrés percibido (EEP)-10.

\begin{tabular}{cccc}
\hline Nivel de EStrés & Puntuaciones & Resultados & VAlor Relativo \\
\hline Nivel bajo & $0-13$ & 12 & $30 \%$ \\
\hline Nivel moderado & $14-26$ & 22 & $55 \%$ \\
\hline Nivel alto & 6 & $15 \%$ \\
\hline Muestra & $27-40$ & 40 & $100 \%$ \\
\hline
\end{tabular}

Fuente: elaboración propia.

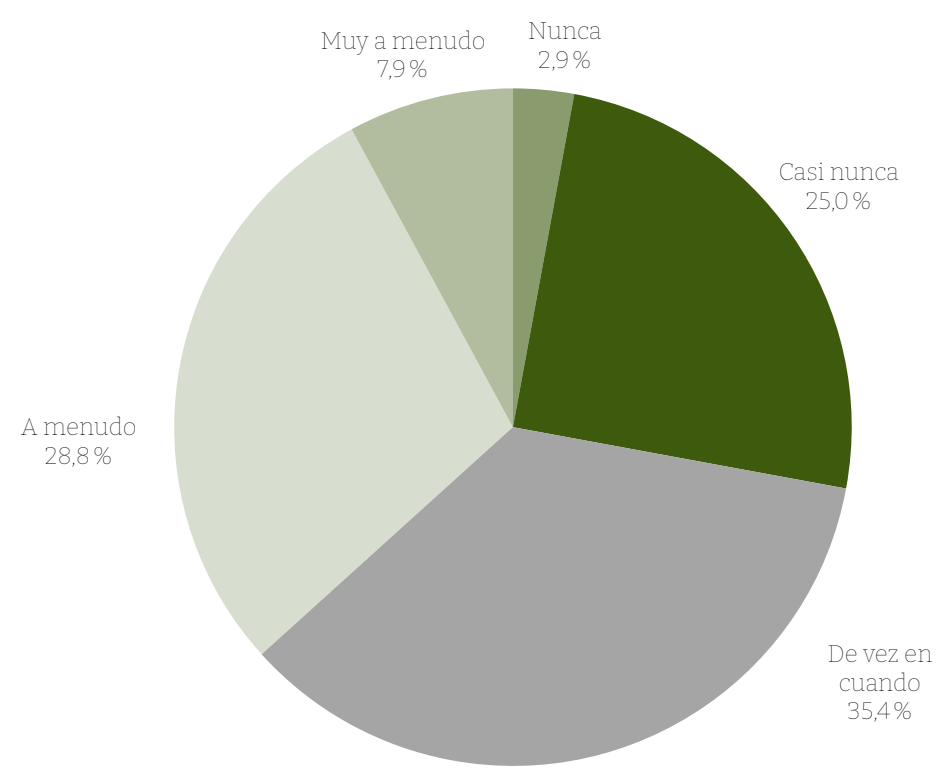

Figura 1. Dimensiones del estrés según ítems 1, 2, 3, 6, 9 y 10.

La presente investigación arroja que el 85\% de los estudiantes de psicología presentan un manejo psicosomático entre moderado y bajo, a casi 3 meses de confinamiento, el estudio presenta una media de 17.98; una mediana de 16.5 y una desviación estándar de 6.86 puntos.

Al observar la frecuencia enlas respuestas de los estudiantes, el 63.3\% manifestó que "de vez en cuando", "casi nunca” o "nunca” se ha sentido afectado o incapaz de afrontar o controlar los problemas o situaciones que se le han presentado en el último mes (dimensión del Estrés), lo que resulta consecuente con la capacidad de afrontamiento, en la que el 58\% respondió que “a menudo"y "muy a menudo" han podido controlar o manejar sus problemas o dificultades.

Según estos resultados, al adelantar la revisión bibliográfica, no se encontraron estudios relacionados con el estrés y su impacto por el confinamiento suscitado por una pandemia, como la causada por la covid-19. Asílas cosas, se analizó el estudio de investigación adelantado por González y Landero (2008) a estudiantes de la Facultad de Psicología de la Universidad Autónoma de Nuevo León, México, que utilizó la escala de estrés percibido (PSS) de 14 ítems, en 


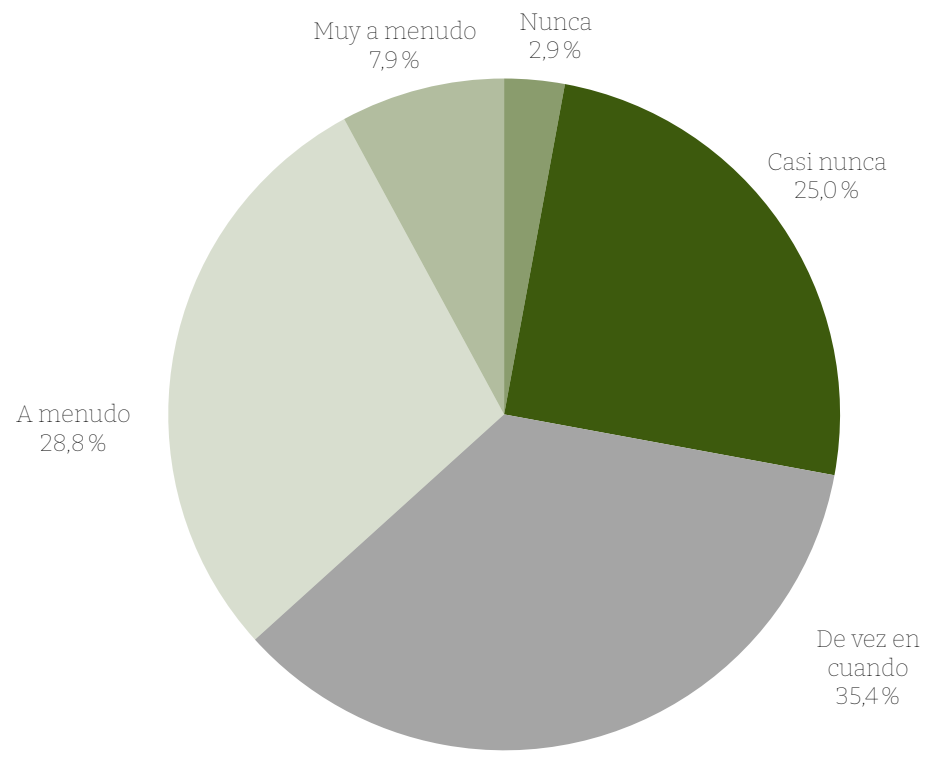

Figura 2. Dimensiones de la capacidad de afrontamiento según ítems 4, 5, 7 y 8.

Fuente: elaboración propia.

2 General Health Questionnaire. la versión adaptada culturalmente a México, la cual presentó una consistencia interna adecuada (coeficiente $\boldsymbol{\alpha}$ de Cronbach = 0.83), con una media de 21.9, una mediana de 21.93 y una desviación estándar de 7.03.

Dicho estudio difiere de la presente investigación, ya que expone a los estudiantes universitarios como una población particular que está expuesta a estresores propios de su cálida estudiantil y a sus efectos, los cuales presentan altos síntomas de estrés debido a las exigencias vitales que sobrepasan sus capacidades personales de afrontamiento. Causa de esto son los diferentes factores académicos que producen reacciones de estrés que, junto al cansancio emocional, se convierten en componentes del desgaste y afectan con su somatización al bienestar percibido.

Con respecto al estrés por confinamiento, como ya fue mencionado, Ruíz (1999) realizó una investigación sobre el estrés en prisión y los factores psicosociales, en la cual concluyó que el ingreso en prisión constituye un evento estresante en muchas ocasiones. Sin embargo, la persona encarcelada se ve expuesta de forma cotidiana a diversas circunstancias que le exigen un esfuerzo de adaptación, como las condiciones de hacinamiento, la misma privación de libertad, la preocupación familiar, las condiciones de equipamiento de los centros y las relaciones interpersonales entre los internos.

Por otra parte, se contrastó el ítem de enfermedad reciente con los indicadores de estrés y del $\mathrm{GHQ}^{2}$ mediante una prueba de comparación de medias para muestras independientes, conla cual se encontró que los sujetos que se sintieron enfermos ( $\mathrm{n}=22$ frente a $\mathrm{n}=30$ de no enfermos) presentan medias más altas en estrés (medias de 2.48 frente 1.99 con $\mathrm{t}=2.07$ y $\mathrm{P}<.05$; la prueba de Levene de homogeneidad de varianzas fue tendencial: $\mathrm{p}=$ Ü.089) y malestar psicológico (2.30 frente a 1.95, $\mathrm{t}=2.21, \mathrm{P}<.05$; prueba de Levene no significativa). Ello mostraba que aquellos sujetos que tenían una percepción subjetiva de estar enfermos mostraban mayores niveles de malestar psicológico.

\section{Conclusiones}

Teniendo en cuenta los antecedentes bibliográficos previamente mencionados frente a la presente investigación, nos lleva a establecer que los estudiantes confinados en la pandemia del Covid-19 han logrado manejar y controlar los estresores que anteriormente los afectaban, algunos de los cuales han desaparecido de manera temporal (estresores ambientales) y los otros se encuentran soportados por familiares y amigos (apoyo social), que son factores fundamental para el afrontamiento de las situaciones que se van presentando. De igual modo, la puntuación en la EEP-10, ubicó al 55\% de los estudiantes encuestados en el rango de "nivel moderado" y el $30 \%$ en el "nivel bajo".

Con base a lo anterior, hace constatar que los estudiantes se encuentran en un nivel moderado en cuanto a la capacidad de afrontamiento, mostrando que, a menudo, en la mayoría de las situaciones saben cómo manejar o afrontar situaciones de estrés a pesar de estar expuestos 
al confinamiento, ya que cuentan con el apoyo familiar en muchas ocasiones y un nivel de confort en casa que, a la par, les permite seguir cumpliendo con sus obligaciones estudiantiles.

En cuanto a la dimensión del estrés, se evidencia que existe un nivel moderado de este, se resalta que los estudiantes de Psicología de la Universidad Santo Tomás pueden llegar a presentar episodios de estrés pero son capaces de asimilarlos y manejarlos, lo cual nos deja la premisa para futuros abordajes sobre qué tan nocivo es a nivel psicológico para los seres humanos esta nueva realidad, ya que los resultados dan cuenta que muchas situaciones de nuestra cotidianidad son más nocivas que lo que estamos atravesando en estos momentos de confinamiento.
Los estudiantes se

encuentran en un nivel

moderado en cuanto a la

capacidad para afrontar

\section{el confinamiento.}

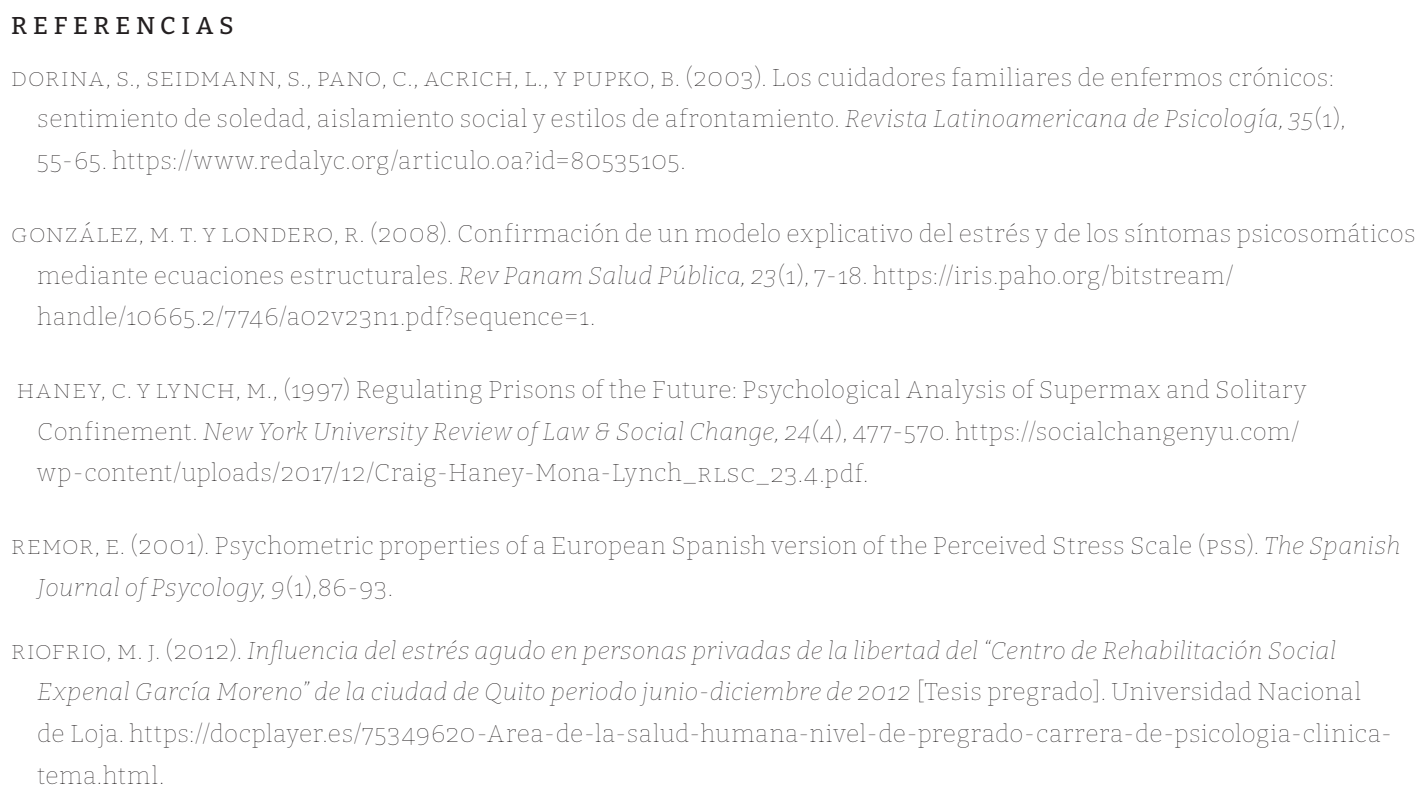

\section{BIBLIOGRAFÍA SUGERIDA}

CAMPO, A., BUSTOS, G., Y ROMERO, A. (2009).Consistencia interna y dimensionalidad de la Escala de Estrés Percibido (EEP-10 y EEP-14) en una muestra de universitarias de Bogotá, Colombia. Aquichán, 9(3), 271-80. https:/www.redalyc org/pdf/741/74112147007.pdf

HERNÁNDEZ, R., FERNÁNDEZ, C., Y BAPTISTA, M. (2014). Metodología de la investigación, sexta edición (6. a ed.). McGraw Hill. ttp://observatorio.epacartagena.gov.co/wp-content/uploads/2017/08/metodologia-de-la-investigacion-sexta-edicion. compressed.pdf 